\title{
PROFILAXIS TROMBOEMBÓLICA EN PACIENTES POSPARTO. ESTUDIO DE CORTE TRANSVERSAL EN TRES INSTITUCIONES EN COLOMBIA
}

\section{Thromboembolic prophylaxis in post-partum patients. Cross-sectional study in three institutions in Colombia}

Jaime Luis Silva-Herrera, $M D^{1}$; Marco Antonio Duque-Giraldo, $M D^{2}$; Camilo Torres-Chaparro, $\mathrm{MD}^{3}$; José Enrique Sanín-Blair, $\mathrm{MD}^{4}$; Magda Lizeth Niño-Peña, MD ${ }^{5}$; Ana María Higuita-Rendón ${ }^{6}$

Recibido: febrero 6/15 - Aceptado: febrero 22/16

\section{RESUMEN}

Objetivo: describir el nivel de riesgo de enfermedad tromboembólica en pacientes posparto y hacer una aproximación al uso actual de la tromboprofilaxis.

Materiales y métodos: estudio descriptivo de corte transversal. Se incluyeron pacientes que fueron hospitalizadas para atención de parto entre agosto de 2012 y abril de 2013 en tres instituciones generales de alta complejidad en Bogotá y Medellín. Se aplicó la Guía de manejo del Royal College Obstetrics \& Gynecology (RCOG) para establecer el riesgo de enfermedad tromboembólica. Las pacientes fueron evaluadas en el posparto y clasificadas en alto, intermedio y bajo riesgo. Se midió la frecuencia real de aplicación de tromboproilaxis. Los resultados se presentan como proporciones.

1 Profesor y jefe, Departamento de Ginecología y Obstetricia, Hospital Universitario San Ignacio, Bogotá (Colombia).jlsilvah1@hotmail.com

2 Jefe, Departamento de Ginecología y Obstetricia, Clínica del Country, Bogotá (Colombia).

3 Coordinador de la Unidad de Medicina Materno Fetal, Clínica del Country, Bogotá (Colombia).

4 Profesor de Ginecología y Obstetricia, Pontificia Universidad Bolivariana, Medellín (Colombia).

5 Residente $3^{\circ}$ año, Ginecología y Obstetricia, Universidad Javeriana, Bogotá (Colombia).

6 Estudiante, Facultad de Medicina Universidad Pontificia Bolivariana, Medellín (Colombia).
Resultados: se evaluaron un total de 3516 pacientes, el $1,9 \%$ de las pacientes fueron clasificadas como de alto riesgo, $42 \%$ de riesgo medio y $56,1 \%$ de riesgo bajo. La tromboprofilaxis solo se aplicó a un $7,4 \%$ de las pacientes con alto riesgo y riesgo intermedio.

Conclusión: según los criterios de la Guía del RCOG, un 43,9\% de las pacientes tendrían indicación de tromboprofilaxis; sin embargo, solo un 7,4\% de ellas la recibió. Se requieren estudios complementarios para evaluar los riesgos y beneficios del uso de la tromboprofilaxis según la guía del Reino Unido.

Palabras clave: tromboembolia venosa, embolia y trombosis, periodo de posparto, prevención y control.

\section{ABSTRACT}

Objective: To describe the risk of thromboembolic disease in post-partum patients and examine the current use of thromboprophylaxis.

Materials and methods: Descriptive crosssectional study that included patients admitted for delivery care between August 2012 and April 2013 in three high-complexity general healthcare institutions in Bogotá and Medellín. The Royal 
College of Obstetrics \& Gynaecology (RCOG) Management Guideline was applied for determining the risk of thromboembolic disease. Patients were assessed during the post-partum period and classified as high, intermediate and low risk. The actual frequency of the use of prophylaxis was measured. Results are presented as proportions.

Results: Overall, 3516 patients were assessed and $1.9 \%$ were classified as high risk, $42 \%$ as intermediate risk and $56.1 \%$ as low risk. Thromboprophylaxis was only used in $7.4 \%$ of patients with high or intermediate risk.

Conclusion: According to the criteria of the RCOG guidelines, $43.9 \%$ of the patients had an indication for thromboprophylaxis and yet only $7.4 \%$ of them received it. Further studies are required to assess the risk and evaluate the benefits and risks of giving thromboprophylaxis in accordance with the United Kingdom guidelines.

Key words: Venous thromboembolism, embolism and thrombosis, post-partum period, prevention and control.

\section{INTRODUCCIÓN}

En Colombia, para el año 2103, las principales causas de mortalidad materna fueron la preeclampsiaeclampsia, la hemorragia posparto, la muerte por causa obstétrica no especificada, la sepsis puerperal y la embolia obstétrica. Respecto a esta última, los eventos trombóticos venosos (ETV) juegan un papel primordial (1). En Estados Unidos, la primera causa de mortalidad materna son los eventos tromboembólicos (ETE) (2).

El embarazo se acompaña por un estado de hipercoagulabilidad que aumenta de tres a cuatro veces el riesgo de ETE, siendo el incremento más marcado en el puerperio (3, 4). El aumento de la coagulabilidad se debe al incremento de factores de coagulación (factor VII, factor VIII, factor de von Willebrand, factor inhibidor del activador del plasminógeno) y a la estasis venosa causada por el embarazo (5).

Los ETE y el embolismo pulmonar (EP) tienen una incidencia en el embarazo de 12,4 x 10000 nacidos vivos y 5,4 x 10000 nacidos vivos respectivamente (6). Una revisión sistemática encontró 12 estudios que describían la incidencia de ETE de origen venoso en el embarazo de 0,82 a 1,98 x 1000 nacidos vivos. Esta revisión halló que once estudios evaluaron la aparición de ETE en el embarazo, seis de ellos mostraron una mayor frecuencia de ETE en el posparto que en el periodo preparto, tres estudios mostraron una menor incidencia en el periodo posparto y dos estudios no encontraron diferencias. La mortalidad asociada al EP varió del 1,1 al 2,4\% (7).

Durante el embarazo y el puerperio se han sido descrito numerosos factores de riesgo para ETV, entre ellos: cesáreas de urgencia, cesáreas electivas, trabajos de parto prolongados, obesidad, inmovilidad, preeclampsia y hemorragia posparto (8). Estos factores, sumados algunos o en forma individual, permiten establecer diferentes niveles de riesgo (9).

El diagnóstico de la ETV está centrado en imágenes diagnósticas como el doppler venoso de miembros inferiores y, en casos de tromboembolismo pulmonar (TEP) en el embarazo, el angio-TAC y la gammagrafía de ventilación-perfusión son considerados los métodos de elección (10).

En la actualidad, existen guías de práctica clínica que abordan el problema de cuándo iniciar tromboprofilaxis en pacientes durante el embarazo y el posparto, para esto estratifican a las gestantes según el riesgo $(9,11)$. En el 2004, la guía del Royal College Obstetrics and Gynecology (RCOG) propuso clasificar las pacientes en riesgo alto, medio o bajo de acuerdo con la presencia de diferentes factores de riesgo. Según esta guía, las pacientes clasificadas en alto riesgo deben recibir tromboprofilaxis durante 6 semanas posparto. Las pacientes clasificadas de riesgo medio deben recibir tromboprofilaxis posparto por 7 días y, finalmente, las pacientes consideradas de bajo riesgo no deben recibir tromboprofilaxis, sino deambulación temprana e hidratación (9). Posterior a la aplicación de la guía del RCOG se redujo la mortalidad materna por ETE en el Reino Unido, de 1,56 x 100000 maternas en el 2004 a 
0,7 x 100000 maternas en el 2008. Adicionalmente, se encontró que el $89 \%$ de estas muertes tenían factores de riesgo que pudieron ser prevenidos (12).

En el medio latinoamericano es escasa la información respecto a la incidencia de enfermedad tromboembólica en el embarazo, como también es poco frecuente la descripción del riesgo de enfermedad trombótica venosa en el embrazo o parto y la descripción de la frecuencia de uso de tromboprofilaxis.

El objetivo de este trabajo es hacer una aproximación a la clasificación del riesgo del RCOG en nuestras pacientes y, con base en esta clasificación, hacer una descripción del uso de tromboprofilaxis en aquellas en las que estaría indicado.

\section{MATERIALES Y MÉTODOS}

Estudio descriptivo de corte transversal. Se incluyeron pacientes con gestaciones mayores a 20 semanas en posparto inmediato, independientemente de su edad, paridad o vía del parto, que consultaron, durante el periodo comprendido entre agosto de 2012 y abril de 2013, a dos instituciones generales de alta complejidad ubicadas en Bogotá (Hospital San Ignacio y Clínica del Country) y Medellín (Clínica Universitaria Bolivariana).

Dos de ellas son hospitales universitarios que atienden población del régimen contributivo en el sistema de seguridad social de Colombia. La tercera es una institución privada que atiende a población del régimen contributivo y seguros de medicina privados. Se realizó un muestreo consecutivo de las pacientes atendidas en el periodo descrito buscando incluirlas a todas.

Procedimiento. Las historias clínicas eran revisadas diariamente a la salida de la paciente por residentes de Ginecología para los hospitales universitarios y por especialistas para el centro privado, con un tiempo protegido de trabajo para su correcta inclusión. Los datos para el cálculo del riesgo se incluyeron en una base de datos específicamente diseñada para este fin (13). Los médicos que ingresaron esta información no conocieron el resul- tado final del nivel de riesgo de cada paciente; a esta información solo tenía acceso el investigador principal del estudio, de manera que no se conoció el resultado de la clasificación de la paciente para definir la terapia tromboprofiláctica durante la hospitalización, con el propósito de no modificar la práctica real al momento del estudio, dado que cada institución manejaba sus propios protocolos en tromboprofilaxis

Los investigadores de cada institución recibieron asesoría para el manejo de la herramienta en una reunión grupal y en forma individual. A cada institución se le asignó un código para ingresar; el investigador principal tenía acceso a la información completa. La guía utilizada para clasificar el riesgo se basa en los criterios descritos por el RCOG2009 (3).

Se midieron los siguientes desenlaces: uso actual de tromboprofilaxis, riesgo de enfermedad tromboembólica venosa, necesidad de terapia tromboprofiláctica.

Análisis. Los resultados se presentan mediante estadística descriptiva; se utilizaron proporciones para describir el nivel de riesgo, el uso de tromboprofilaxis actual y la proporción de pacientes que la recibieron en relación con las que la habrían debido recibir según la guía del RCOG.

El protocolo fue presentado y aprobado por los comités de ética e investigación de cada institución.

\section{RESULTADOS}

De un total de 6795 partos, se incluyeron 3516 pacientes en las tres instituciones (51,7\%); el mayor número de pacientes provino del hospital San Ignacio (69,3\%) (figura 1).

La terapia farmacológica como manejo de tromboprofilaxis se le aplicó al 7,4\% de las pacientes.

Las pacientes fueron clasificadas según el riesgo de enfermedad tromboembólica de la siguiente manera: el 1,9\% de alto riesgo, el $42 \%$ de riesgo medio y 56,1\% de riesgo bajo. El número de pacientes por nivel de riesgo y por institución se presenta en la tabla 1. 
Figura 1.

\section{Flujograma de pacientes}

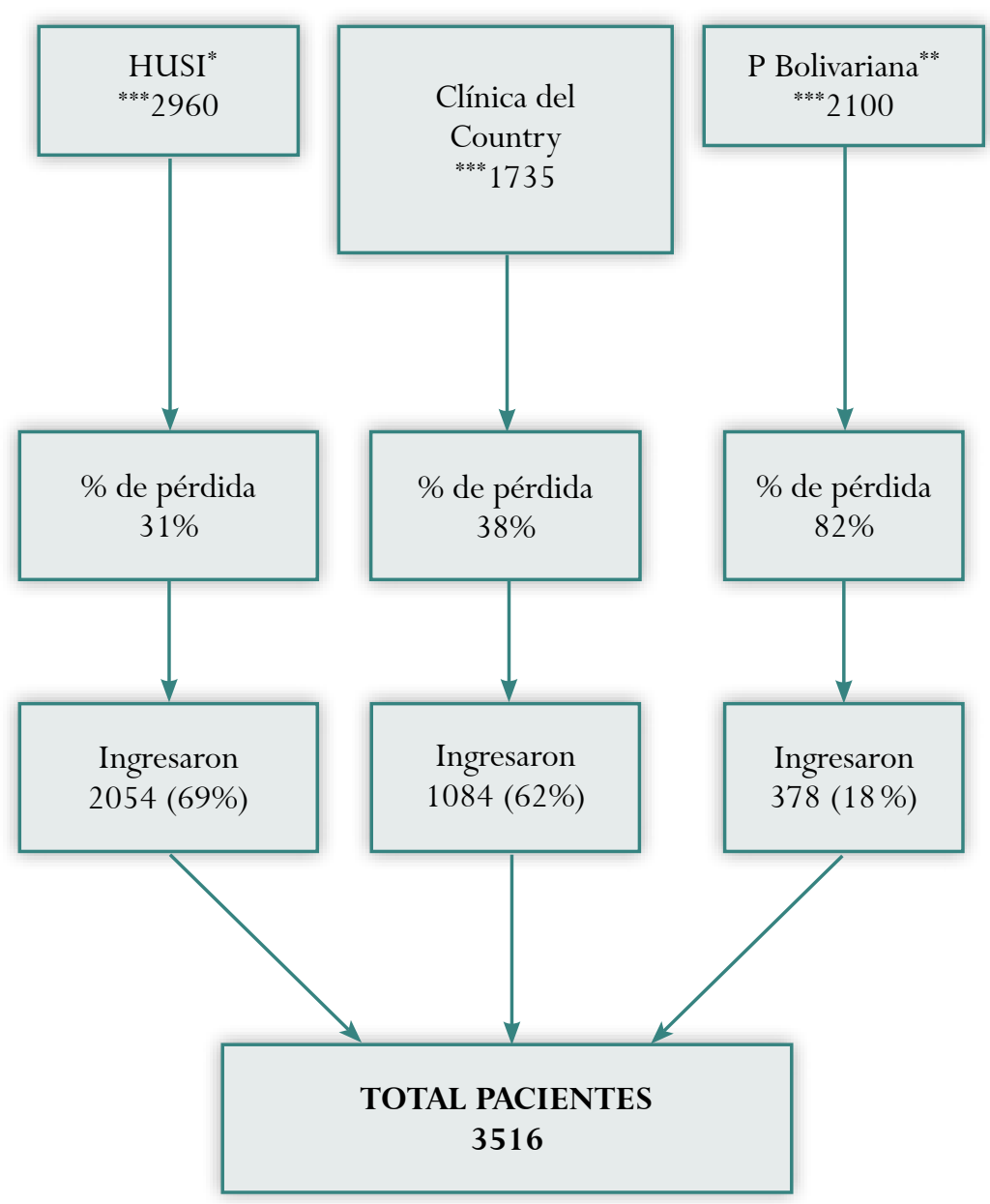

*HUSI: Hospital Universitario San Ignacio.

${ }^{* *}$ P. Bolivariana: Clínica Pontificia Bolivariana

**** Número de partos atendidos en el periodo de tiempo del estudio en cada institución.

Según la recomendación del RCOG, el 43,9\% de las pacientes deberían haber recibido tromboprofilaxis.

\section{DISCUSIÓN}

Este estudio presenta una frecuencia de alto riesgo de ETE en el posparto (1,9\%) y de riesgo medio (42\%). También, una posible subutilización de la profilaxis de enfermedad tromboembólica en pacientes obstétricas a la luz de la recomendación hecha por la guía del RCOG: del 43,9\% que fueron calificadas como de riesgo alto o medio, por lo que habrían debido recibir profilaxis, solo la recibieron el 7,4\% según la práctica actual.
Hay pocos estudios disponibles acerca de la distribución de la frecuencia de la clasificación del riesgo según la guía del RCOG. En Irán se informa que mediante la clasificación sugerida por la primera versión de la guía publicada en el 2004, el $40 \%$ tenía indicada la tromboprofilaxis, dato ligeramente menor al reportado en este estudio (14).

En cuanto al manejo de las pacientes con riesgo incrementado de ETE en un estudio realizado en Brasil, en una cohorte de 27000 pacientes, se informa que hasta el $25 \%$ de aquellas que fueron clasificadas de alto riesgo y el $45 \%$ de riesgo moderado de ETE no recibieron tromboprofilaxis 


\begin{tabular}{|l|c|c|c|c|}
\hline \multicolumn{5}{|c|}{ Cabla 1. } \\
\begin{tabular}{|l|c|c|c|}
\hline \multicolumn{4}{|c|}{ Clasificación del riesgo de enfermedad tromboembólica en mujeres } \\
en posparto en tres instituciones en Colombia
\end{tabular} \\
\hline Institución/Riesgo & Riesgo alto & Riesgo medio & Riesgo bajo & Total \\
\hline Hospital Universitario San Ignacio & $35(1,7 \%)$ & $944(45,9 \%)$ & $1075(52,3 \%)$ & 2054 \\
\hline Clínica del Country & $28(2,5 \%)$ & $467(43 \%)$ & $589(54,3 \%)$ & 1084 \\
\hline Clínica Universitaria Bolivariana & $6(1,5 \%)$ & $139(36,7 \%)$ & $233(61,6 \%)$ & 378 \\
\hline
\end{tabular}

para el momento del estudio (15). Por otra parte, Alexander et al. (16) evaluaron en Estados Unidos un total de 2693760 pacientes con partos vaginales entre 2006 y 2012. Un 60\% de las pacientes con trombofilia y un $74 \%$ de quienes tenían antecedente de ETE, consideradas de alto riesgo, recibieron tromboprofilaxis. En pacientes con factores de riesgo como obesidad o enfermedad cardiaca, solo la recibieron cerca del 6\%. De esta manera se evidencia, en la práctica, un cubrimiento muy inferior al recomendado.

Parte de la dificultad en establecer la frecuencia de los diferentes niveles de riesgo en las poblaciones podría obedecer al hecho de que se dispone de múltiples guías de manejo para ETE (17), muchas de las cuales comparten los criterios o factores de riesgo del RCOG, pero la cuantificación de este ha sido hecha con mediciones diferentes. Esta disparidad se puede explicar porque la evidencia para apoyar o sugerir diferentes factores de riesgo como indicativos de tromboprofilaxis no es alta (17). Un trabajo publicado por Palmerola et al., en el que se comparan tres guías con 293 pacientes sometidas a cesárea encontraron que el $85 \%$ de las pacientes, según el RCOG, deberían recibir tromboprofilaxis, el 1\% según recomendaciones de ACOG y el 38,4\% según las guías del CHEST (18). No obstante, existe una constante en todas las guías que es importante resaltar y es la necesidad de evaluar el riesgo trombótico en todas las pacientes durante el embarazo y en el posparto inmediato (16).

La investigación tiene limitaciones derivadas de un alto sesgo de selección dado que no se incluyó el $48 \%$ de las pacientes que hacían parte de la pobla- ción de estudio, no se estudiaron las características basales de la población estudiada como tampoco de aquella que no ingresó en el estudio.

Como fortaleza se tiene que es la primera aproximación que se hace a la evolución del riesgo de ETE en Colombia y que describe el uso actual de la tromboprofilaxis en nuestras instituciones. Llama la atención sobre la necesidad de nuevos estudios que describan la magnitud del problema y sus consecuencias

\section{CONCLUSIÓN}

Se requiere la estadificación del riesgo de ETE en el embarazo y el posparto para poder definir mejor las candidatas a tromboprofilaxis. Es posible que haya un importante subtratamiento de las pacientes con riesgo alto y mediano de ETE en el medio local.

\section{REFERENCIAS}

1. Departamento Nacional de Estadística (DANE). Estadísticas vitales. Muertes no fetales. Defunciones maternas por grupo de edad y causa [visitado 2016 Ene 21]. Disponible en: http://www.dane.gov.co/ index.php/esp/poblacion-y-demografia/nacimientos-y-defunciones/118-demograficas/estadisticasvitales/4675-defunciones-no-fetales-2013

2. Donnelly JC, D'Alton ME. Pulmonary embolus in pregnancy. Semin Perinatol. 2013;37:225-33.

3. Royal College of Obstetricians and Gynaecologists. Thromboembolic Disease in Pregnancy and the Puerperium: Acute Management. Clinical Guideline. Green-top Guideline 28. London: RCOG; 2009.

4. James AH, Grotegut CA, Brancazio LR, Brown H. Thrombomebolism in pregnancy: recurrence and its prevention. Semin Perinatol. 2007;3:167-75. 
5. The American College of Obstetrics and Gynecologist. Thromboembolism and Pregnancy. Practice Bulletin; 2011. p. 123.

6. SOGC Clinical Practice Guideline. Venous Thromboembolism and anti-thrombotic therapy in pregnancy. JOCG. 2014;308.

7. Kourlaba G, Relakis J, Kontodimas S, Holm MV, Maniadakis N. A systematic review and metaanalysis of the epidemiology and burden of venous thromboembolism among pregnant women. Int J Gynaecol Obstet. 2016;132:4-10.

8. Rodger M. Pregnancy and venous thromboembolism: 'TIPPS' for risk stratification. Hematology Am Soc Hematol Educ Program. 2014:387-92.

9. Royal College of Obstetricians and Gynaecologists. Reducing the risk of venous thromboembolism during pregnancy and the puerperium. Green Top Guideline No. 37; 2015.

10. Durán-Mendicutti A, Sodickson A. Imaging evaluation of the pregnant patient with suspected pulmonary embolism. Int J Obstet Anesth. 2011;20:51-9.

11. Bates SM, Greer IA, Middeldorp S, Veenstra DL, Prabulos AM, Vandvik PO. VTE, thrombophilia, antithrombotic therapy, and pregnancy: Antithrombotic Therapy and Prevention of Thrombosis. 9th ed. American College of Chest Physicians Evidence-Based Clinical Practice Guidelines. Chest. 2012;141:e691S$736 \mathrm{~S}$.
12. Centre for Maternal and Child Enquiries (CMACE). Saving Mothers' Lives: reviewing maternal deaths to make motherhood safer: 2006-08. The Eighth Report on Confidential Enquiries into Maternal Deaths in the United Kingdom. BJOG. 2011;118(Suppl 1):1-203.

13. Fundación Santafé de Bogotá. Centro de Innovación e investigación en salud. Zona libre de trombosis [visitado 2016 Feb 12]. Disponible en: https://www. zonalibredetrombosis.org/

14. Cregan A, Higgins JR, O'Shea S. Implementation of thromboprophylaxis guidelines. Ir Med J. 2013; 106:80-2.

15. Caiafa JS, de Bastos M, Moura LK, Raymundo S. Brazilian Registry of venous thromboembolism prophylaxis. Managing venous thromboembolism in Latin American patients: emerging results from the Brazilian Registry. Semin Thromb Hemost. 2002;28 Suppl 3:47-50.

16. Friedman AM, Ananth CV, Prendergast E, Chauhan SP, D'Alton ME, Wright JD. Thromboembolism incidence and Prophylaxis during vaginal delivery hospitalizations. Am J Obstet Gynecol. 2015;212:221.e1-12.

17. Okoroh EM, Azonobi IC, Grosse SD, Grant AM, Atrash HK, James AH. Prevention of venous thromboembolism in pregnancy: a review of guidelines, 20002011. J Womens Health (Larchmt). 2012;21:611-5.

18. Palmerola K, D’alton M. A comparison of recommendations for pharmacologic thromembolism prophylaxis after cesarean delivery from three major guidelines. BJOG.2015; DOI: 10.1111/1471-0528.13706.

Conflicto de intereses: para la elaboración de este estudio se utilizó la página web: www. Zonalibre de trombosis.org, la cual es una herramienta de evaluación de la calidad diseñada por el laboratorio Sanofi-Aventis. Sanofi-Aventis no participó en el diseño, la elaboración, la tabulación ni en las conclusiones de este estudio; agradecemos su apoyo y colaboración. 